\title{
Statistical Learning and Inference Is Impaired in the Nonclinical Continuum of Psychosis
}

\author{
${ }^{\circledR}$ Ilvana Dzafic, ${ }^{1,2,3,4}{ }^{\circledR}$ Roshini Randeniya, ${ }^{2}$ Clare D. Harris, ${ }^{\circledR}{ }^{\circledR}$ Moritz Bammel, ${ }^{5}$ and ${ }^{\circledR}$ Marta I. Garrido ${ }^{1,2,3,4}$ \\ ${ }^{1}$ Melbourne School of Psychological Sciences, University of Melbourne, Parkville 3010, Victoria, Australia, ${ }^{2}$ Queensland Brain Institute, University \\ of Queensland, Brisbane 4072, Queensland, Australia, ${ }^{3}$ Australian Research Council Centre of Excellence for Integrative Brain Function, Clayton \\ 3800, Victoria, Australia, ${ }^{4}$ Centre for Advanced Imaging, University of Queensland, Brisbane 4072, Queensland, Australia, and ${ }^{5}$ Institute of \\ Cognitive Science, University of Osnabrueck, 49074 Osnabrueck, Germany
}

Our perceptions result from the brain's ability to make inferences, or predictive models, of sensory information. Recently, it has been proposed that psychotic traits may be linked to impaired predictive processes. Here, we examine the brain dynamics underlying statistical learning and inference in stable and volatile environments, in a population of healthy human individuals $(N=75$; 36 males, 39 females) with a range of psychotic-like experiences. We measured prediction error responses to sound sequences with electroencephalography, gauged sensory inference explicitly by behaviorally recording sensory statistical learning errors, and used dynamic causal modeling to tap into the underlying neural circuitry. We discuss the findings that were robust to replication across the two experiments (Discovery dataset, $N=31$; Validation dataset, $N=44$ ). First, we found that during stable conditions, participants demonstrated greater precision in their predictive model, reflected in a larger prediction error response to unexpected sounds, and decreased statistical learning errors. Moreover, individuals with attenuated prediction errors in stable conditions were found to make greater incorrect predictions about sensory information. Critically, we show that greater errors in statistical learning and inference are related to increased psychotic-like experiences. These findings link neurophysiology to behavior during statistical learning and prediction formation, as well as providing further evidence for the idea of a continuum of psychosis in the healthy, nonclinical population.

Key words: inference; prediction error; psychosis continuum; statistical learning; volatility

\section{Significance Statement}

While perceiving the world, we make inferences by learning the statistics present in the sensory environment. It has been argued that psychosis may emerge because of a failure to learn sensory statistics, resulting in an impaired representation of the world. Recently, it has been proposed that psychosis exists on a continuum; however, there is conflicting evidence on whether sensory learning deficits align on the nonclinical end of the psychosis continuum. We found that statistical learning of sensory events is associated with the magnitude of mismatch negativity and, critically, is impaired in healthy people who report more psychotic-like experiences. We replicated these findings in an independent sample, demonstrating strengthened credibility to support the continuum of psychosis that extends into the nonclinical population.

\section{Introduction}

Sensory perception is facilitated by prior beliefs about what is likely to happen next (Sherman et al., 2015). By estimating the probability of events given the history, we can form a predictive

Received Feb. 11, 2020; revised June 23, 2020; accepted June 30, 2020.

Author contributions: I.D. and M.I.G. designed research; I.D., C.D.H., and M.B. performed research; I.D. and R.R. analyzed data; I.D. wrote the paper.

The authors declare no competing financial interests.

This work was funded by the Australian Research Council Centre of Excellence for Integrative Brain Function (ARC Centre Grant CE140100007) and a University of Queensland Fellowship (2016000071) to M.I.G., as well as a University of Queensland International Research Scholarship to R.R. We thank the participants for their time.

Correspondence should be addressed to Ilvana Dzafic at ilvana.dzafic@unimelb.edu.au.

https://doi.org/10.1523/JNEUROSCI.0315-20.2020

Copyright $\odot 2020$ the authors model about the world. When circumstances are "volatile," such that previously learned regularities change, it is advantageous to form more flexible predictive models (Mathys et al., 2011). Indeed, previous literature has shown that healthy individuals are able to estimate environmental volatility (Behrens et al., 2007), adopting a greater learning rate in the face of ever changing, volatile circumstances. This motivates exploratory behavior and continuous updating, as well as suppressing of top-down prior beliefs (Angela and Dayan, 2002).

The state of constant learning is inefficient as a long-term strategy in environments that are stable (Schwartenbeck et al., 2013). In a stable environment, sensory information varies less. Consequently, the precision (mathematically speaking, the inverse of variance) of prior beliefs is greater, and these beliefs 
thereby influence perception more strongly. This greater reliance on prior beliefs reduces the cognitive demands associated with perception (Vossel et al., 2014). In these environments, healthy individuals form precise predictions about forthcoming sensory stimuli, and their brains consequently produce large prediction error (PE) responses to events that violate such predictions (Wacongne et al., 2012).

A sensory PE response is commonly gauged using electroencephalography (EEG) and an auditory oddball paradigm (Squires et al., 1975), in which surprising sounds are embedded in a sequence of predictable sounds. Within this paradigm, the predictable sounds (standards) are subtracted from the surprising sounds (deviants) to obtain the mismatch negativity (MMN) event-related potential (ERP), which is interpreted as the neural manifestation of a failure to suppress a PE (Garrido et al., 2009a). The $\mathrm{PE}$ response more generally refers to the difference between deviants and standards, whereas ERP components, such as the $\mathrm{MMN}$, refer to a specific peak/time window. PE responses are thought to signify implicit sensory learning ability: an individual's accuracy in their inherent learning of the statistics of sensory events (Lecaignard et al., 2015; Southwell and Chait, 2018). However, it has not as yet been addressed whether MMN scales with the accuracy in statistical learning and inference.

Emerging theoretical accounts of psychosis postulate that psychotic experiences arise because of an impairment in the predictive ability of the brain to infer the causes of internal and external sensations (Adams et al., 2016; Sterzer et al., 2018). The most robust and replicable empirical evidence for this is from findings of attenuated PE responses in psychosis (Adams et al., 2013; Näätänen et al., 2015). Alterations in the neurophysiology of PEs have been shown to increase as psychotic traits increase, suggesting that the degree of $\mathrm{PE}$ attenuation aligns on a continuum of psychosis (Randeniya et al., 2018). The psychosis continuum comprises the full spectrum of psychotic experiences, from healthy individuals who report psychotic-like experiences to those with florid psychosis at the very end of the spectrum (van Os and Reininghaus, 2016). In this study, we zoom in to the nonclinical population, as there is conflicting evidence about whether PEs are also reduced on the healthy end of the continuum (Broyd et al., 2016; Oestreich et al., 2019).

The aims of this study were to examine the relationship among statistical learning behavior, sensory PEs, the ability of the brain to attune to volatility, and to investigate whether aberrations in these are aligned on the nonclinical continuum of psychosis. In response to the current replication crisis in the field, we sought to corroborate our findings in an independent sample. In so doing, we hoped to assess the robustness of our initial findings, using the same methodology in an unbiased manner. In other words, we ensured that both preprocessing and analysis choices were not biased by the original findings. To this end, we report the findings side-by-side for both datasets and discuss the implications of the replicated findings.

\section{Materials and Methods \\ Participants}

The total sample from both the Discovery and Validation study included 75 healthy adults. The Discovery study included 31 participants (age range, $19-38$ years; mean age, 24.65 years; $\mathrm{SD}=4.85 ; 14$ males and 17 females), and the Validation study included 44 participants (age range, 18 -39 years; mean age, 24.27 years; $S D=5.13 ; 22$ males and 22 females). For the Discovery study, a power analysis was performed (R software: "pwr.r.test" function), yielding a sample size of $N=28$ for $80 \%$ power to observe moderate effects at $\alpha=0.05$, two-sided. Moderate effect sizes
Table 1. Demographic information

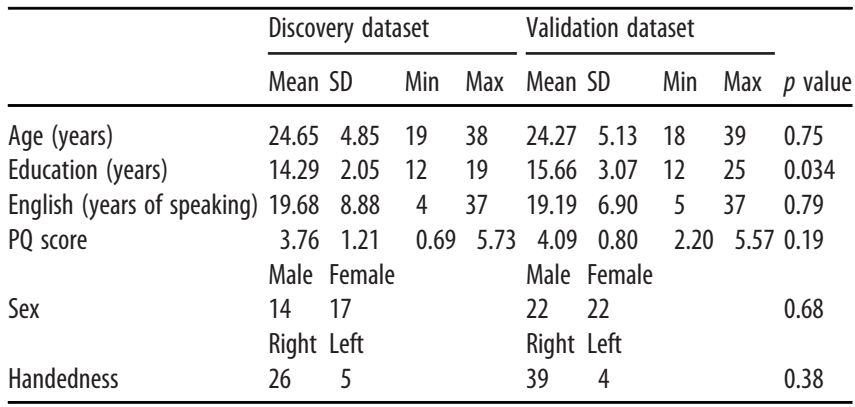

Max, Maximum; Min, minimum. PQ Psychotic experience scores are log transformed. Missing information from one Validation study participant for education, English, and handedness.

have been reported in a previous study investigating auditory oddball ERP correlates of schizotypal perceptual alterations (Jang and Kim, 2014; Sumich et al., 2014). For the Validation study, we decided to increase our power to $95 \%$, which yielded a sample size of $N=45$ to observe a moderate effect at $\alpha=0.05$, two-sided. We did collect 45 participants originally; however, one of these participants reported that the sounds in the task had intermittently stopped playing, so we removed them from all of the final analyses.

All participants were recruited through the Psychology Research Participation Scheme (SONA Database), an online newsletter to staff and students across the University of Queensland and Gumtree. Prior screening confirmed that all participants did not have a history of psychiatric or neurologic disorders, and were not currently taking medication or using any illicit drugs. The highest level of education, smoking habits, and alcohol consumption were recorded. Participants also completed the 92-item Prodromal Questionnaire (PQ), which measures positive and negative symptoms and is typically used to assess psychotic experiences in healthy individuals (Loewy et al., 2005). For further information on the demographics of the sample and the PQ scores per participant, as well as the positive and negative symptom frequencies, please see Table 1 and Figure 1. Participants provided written informed consent for taking part in our study after reading and understanding the information sheet, which included a full description of the study and procedure. Participants received monetary reimbursement for their time. Participant recruitment and data collection for the Discovery and Validation samples were conducted by independent researchers in the same laboratory using the same methodology. This research was approved by the University of Queensland Human Research Ethics Committee.

\section{Experimental design}

Reversal oddball task. An auditory "duration" oddball paradigm was modified so that the probability of different sounds varying in duration was either stable or volatile (adapted from Weber et al., 2018). In a stable experimental block, a particular sound was always more likely than another sound (e.g., short sounds had $80 \%$ probability, long sounds had $20 \%$ probability, and vice versa). In volatile experimental blocks, a particular sound, which was more likely at first, was then less likely, with three reversals of probability in each block. The Reversal oddball task is represented in Figure 2.

Auditory stimuli and design. The Reversal oddball paradigm consisted of 2000 pure tones played over eight experimental blocks ( 2 min 8 $\mathrm{s}$ each; four stable and four volatile blocks). The tones varied in duration such that short tones lasted $50 \mathrm{~ms}$ and long tones lasted $100 \mathrm{~ms}$. All tones had an identical frequency of $500 \mathrm{~Hz}$ and smooth rise and fall periods of $5 \mathrm{~ms}$. The tones were presented in a pseudorandom order, with each presentation of five tones including a deviant tone in a randomly assigned position; the deviant tones were always separated by at least one standard tone. The tones were delivered binaurally via insert earphones for $\sim 2$ min every $500 \mathrm{~ms}$. Sound intensity was kept constant between participants at a comfortable level. The short sounds $(50 \mathrm{~ms})$ and long sounds $(100 \mathrm{~ms})$ were both played 1000 times each throughout the experiment. In the entire experiment, we had the following: two stable blocks in 


\section{Discovery Dataset}

A

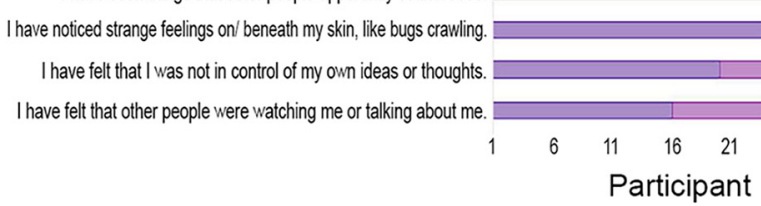

I have felt like I was at a distance from myself, as if I were outside my own body or that a part of my body did not belong to me. I have felt unable to enjoy things that I used to enjoy. I have found it hard to be emotionally close to other people. I have had nothing to say or very little to say.

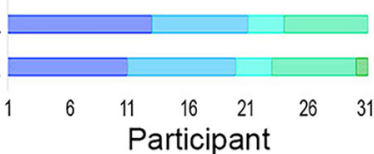

B

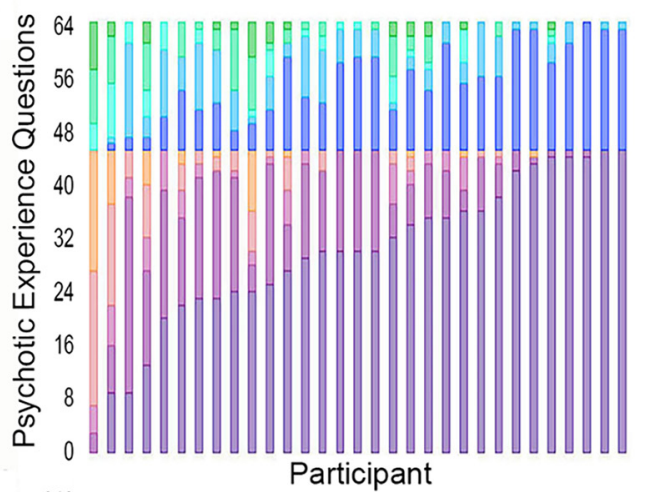

C

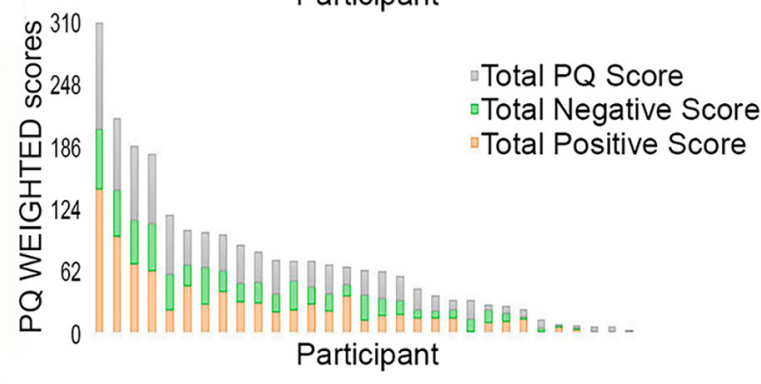

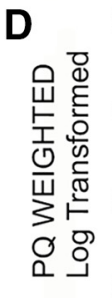

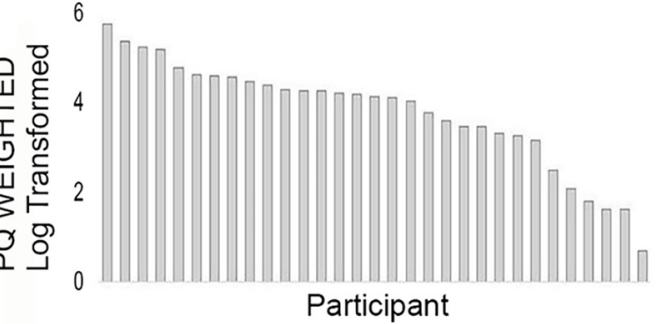

\section{Validation Dataset}

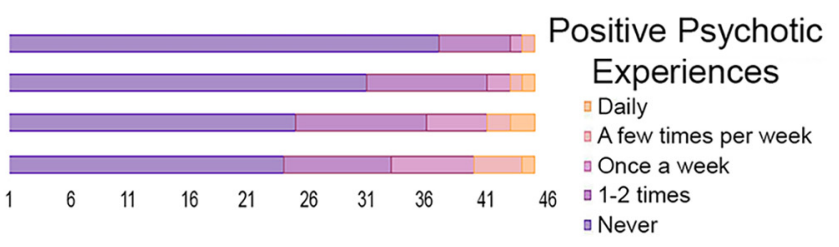

Negative Psychotic Experiences

$\llbracket$ Daily

$\square$ A few times per week - Once a week घ1-2 times

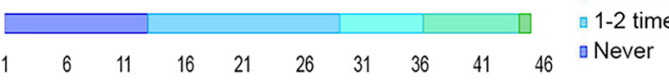

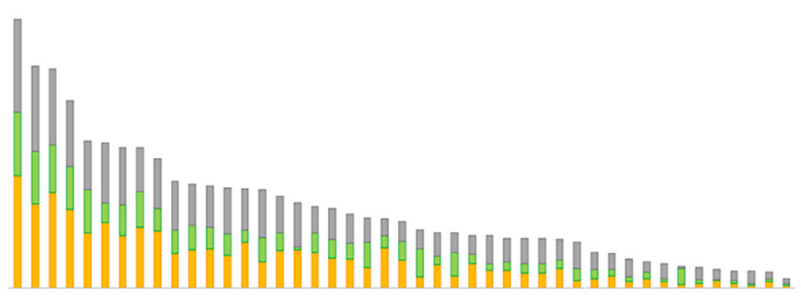

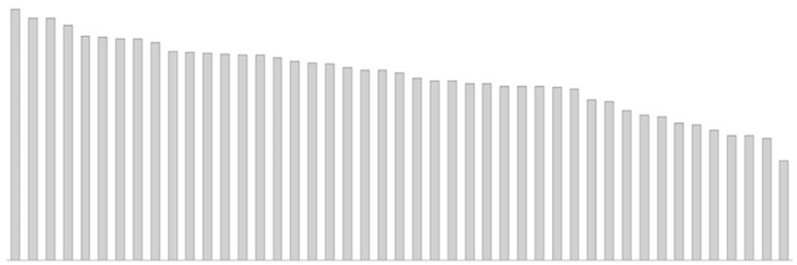

Figure 1. $\mathrm{PQ}$ scores for Discovery and Validation samples. $\boldsymbol{A}$, Frequency of a subset of positive psychotic experiences (purple to orange) and negative psychotic experiences (blue to green). $\boldsymbol{B}$, Frequency of psychotic experiences, displaying positive psychotic experiences (purple to orange) and negative psychotic experiences (blue to green). C, PQ scores weighted by frequency, displaying total PQ weighted scores (gray), negative weighted scores (green), and positive weighted scores (orange). D, Log-transformed PQ-weighted scores.

which the short sound was more probable (at $80 \%$ ); two stable blocks in which the long sound was more probable (at $80 \%$ ); and four volatile blocks in which each block had two times where the short sounds were more probable (at $80 \%$ ) and also two times where the long sounds were more probable (at $80 \%$ ). The order of stable and volatile blocks was counterbalanced across participants.

Procedure. During the Reversal oddball task, participants were seated on a comfortable chair in front of a desk and computer screen, in a dimly lit Faraday cage testing room. Before the experiment, the participants were familiarized with the different sound types and trained with two short practice runs of the task. Participants were asked not to move while the sounds were played and to look at a fixation cross at the center of the screen. The participants were instructed to pay attention to the sounds to judge the probability of the more likely sound and rate their confidence on this judgment. Probability was rated on a scale (Fig. 2); the left end of the scale represents $100 \%$ short sounds and the right end of the scale represents $100 \%$ long sounds. Confidence in probability estimation was on a scale of $1=$ not confident, $2=$ moderately confident, and $3=$ very confident. Participants were required to make these estimates every 2 min and 8 s using a computer keyboard and a mouse. The total duration of the Reversal oddball task was $\sim 20$ min (including short breaks). 
Q : Which sound did you hear more often?
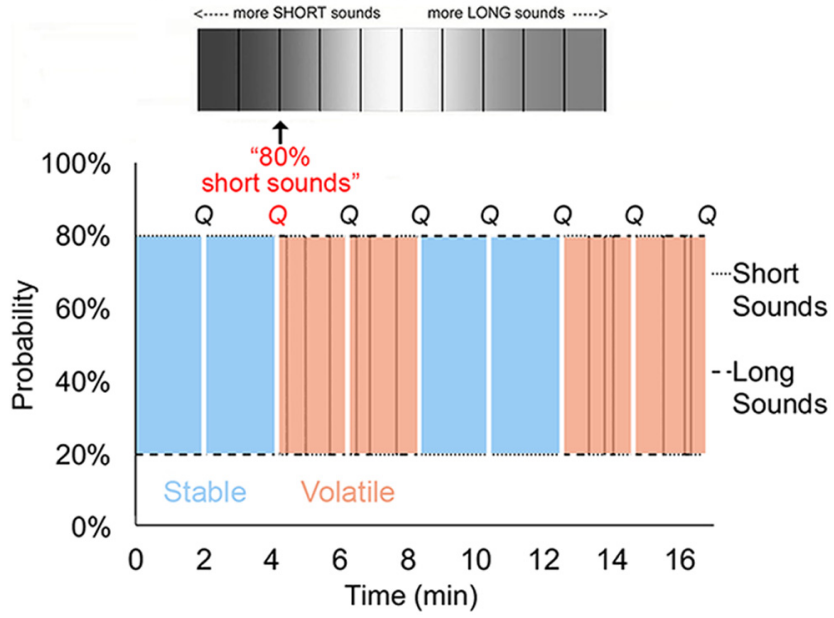

Figure 2. Reversal oddball task. A schematic diagram showing an example of an experimental run. The order of the blocks in an experimental run was counterbalanced between participants. In the first stable block depicted here, the short sound $(50 \mathrm{~ms})$ was more probable (80\%) throughout, whereas in the first volatile block the long sound $(100 \mathrm{~ms})$ was more probable at first, then the short sound was more probable, with three reversals (vertical lines) of probability in total in each volatile block. Participants were asked to listen to the sounds and estimate the probability (top $Q$ ) of the most frequent sound and rate their confidence on this judgment every $2 \mathrm{~min}$ and $8 \mathrm{~s}$.

EEG recording and preprocessing. A BioSemi Active Two System recorded continuous EEG data from 64 scalp electrodes at a sampling rate of $1024 \mathrm{~Hz}$. Electrodes were arranged according to the international 10-20 system for electrode placement (Oostenveld and Praamstra, 2001). Standard preprocessing and data analysis were performed with SPM12 (http://www.fil.ion.ucl.ac.uk/spm/). Data were referenced to standard BioSemi reference electrodes, downsampled to $200 \mathrm{~Hz}$ and high-pass filtered at $0.5 \mathrm{~Hz}$ using the Butterworth filter. Eye blinks were detected and marked using the VEOG (vertical EOG) channel at an eyeblink threshold of 4; the Berg method was used to correct for eye blinks. The data were epoched offline with a peristimulus window of -100 to $400 \mathrm{~ms}$. Further artifact rejection was performed by thresholding all channels at $100 \mu \mathrm{V}$, robustly averaging across trials, applying a low-pass Butterworth filter of $40 \mathrm{~Hz}$, and baseline correcting between -100 and $0 \mathrm{~ms}$. We analyzed event-related potentials from the onset of standard and oddball tones separately for stable and volatile conditions.

\section{Statistical analysis}

In the current study, we used both frequentist and Bayesian approaches to our analyses. The Bayesian analyses were conducted using the JASP package (https://jasp-stats.org/). The frequentist analyses were conducted using the SPSS package (2012 version; IBM); multiple correlations were corrected using the method of Sidak (1967). In the Validation study, we excluded two participants in the neuroimaging analyses because of EEG trigger failure and high impendence (more than $\pm 50 \Omega$ ). Further, one participant was removed from the ERP analyses, and one participant from the PQ analyses, as they were an outlier ( $z$ score, greater than \pm 3 ).

Bayesian approach. Bayes factors are based on Bayes' rule, displayed in the equation below. The posterior probability, given the observed data (the posterior; $p\left(H_{1} \mid\right.$ data $) / p\left(H_{0} \mid\right.$ data $\left.)\right)$, equals the prior odds [the odds of the null and alternative hypotheses $\left(p\left(H_{1}\right) / p\left(H_{0}\right)\right)$ before the data are observed], multiplied by the Bayes factor $\left(p\left(\right.\right.$ data $\left.\mid H_{1}\right) / p\left(\right.$ data $\left.\left.\mid H_{0}\right)\right)$, or the change (update) from before the posterior (Wagenmakers et al., 2017), as follows:

$$
\underbrace{\frac{p\left(H_{1} \mid \text { data }\right)}{p\left(H_{0} \mid \text { data }\right)}}_{\text {The Posterior }}=\underbrace{\frac{p\left(H_{1}\right)}{p\left(H_{0}\right)}}_{\text {The Prior }}=\underbrace{\frac{p\left(\text { data } \mid H_{1}\right)}{p\left(\text { data } \mid H_{0}\right)}}_{\text {Bayes factor } \mathrm{BF}_{10}} .
$$

The subscript " 10 " in $\mathrm{BF}_{10}$ indicates that in the equation $H_{1}$ (the alternative hypothesis) is in the numerator and $H_{0}$ (the null hypothesis) is in the denominator, and subscript "01" indicates the reverse. In the current study, Bayesian factors were computed using $\mathrm{BF}_{10}$, which indicates testing the alternative hypothesis over null hypothesis. We report on $\mathrm{BF}_{-0}$ and $\mathrm{BF}_{+0}$, which indicate that the alternative hypothesis specifies that the correlation is either negative $\left(_{-0}\right)$ or positive $\left(_{+0}\right.$; Gronau et al., 2020).

We conducted single-channel ERP, whole-channel spatiotemporal, source-level, and effective connectivity analyses to assess the effect of environment (stable vs volatile) on neuronal activity. In addition, we conducted behavioral analyses to investigate statistical learning in the different volatility contexts, and its associations with psychotic-like experiences and neural dynamics. We have described these analyses in more detail below.

Single-channel analysis. We conducted a full factorial $2 \times 2$ withinsubjects ANOVA design on mean ERP values, with Environment (stable and volatile) and MMN (Standard and Deviant) as factors. Mean ERP values were obtained by identifying the maximum amplitude across the preselected time window of interest, which is typical for MMN latency, as follows: $150-250 \mathrm{~ms}$, over a frontocentral channel $(\mathrm{Fz})$ in which MMN responses are typically seen in oddball paradigms (Todd et al., 2008). We contrasted evoked responses to deviant and standard sounds, under stable and volatile conditions. Significant interactions were further analyzed using paired $t$ tests.

Behavioral analysis. We conducted paired $t$ tests on mean statistical learning errors and mean confidence scores in stable versus volatile conditions. This was done to assess the effect of environment (stable vs volatile) on statistical learning and confidence in estimating probabilities. Statistical learning errors are here defined as the absolute percentage errors in probability estimation; that is, the difference between the participants' probability estimation of the more likely sound and the actual probability of the more likely sound. Thus, higher statistical learning error values reflect less accurate statistical learning. Next, we computed Pearson's and Bayesian correlations to assess the associations among psychotic experience, statistical learning errors, and $\mathrm{MMN}$ in stable and volatile conditions.

Spatiotemporal maps and source reconstruction. Three-dimensional spatiotemporal images were generated from averaged ERP data for each participant and condition. A two-dimensional matrix corresponding to the scalp electrode space was produced for each time bin from 0 to $400 \mathrm{~ms}$ in steps of $5 \mathrm{~ms}$. The images were assembled according to their peristimulus temporal order, which resulted in a three-dimensional spatiotemporal image $(32 \times 32 \times 81)$ per participant. These images were then smoothed at a full-width at half-maximum of $12 \times 12 \times 20 \mathrm{~ms}$. In addition, we performed source reconstruction of the spatiotemporal image volumes to make inferences about the cortical regions that generated the scalp data. We coregistered the sensor data with a single sphere head model to obtain the source estimates on the individuals' cortical mesh. Next, we conducted forward computations of the effect each dipole on the cortical mesh has on the sensors. Finally, we inverted the forward computations with the multiple sparse priors algorithm under group constraints (Friston et al., 2008; Litvak and Friston, 2008); these inverse reconstructions were summarized as images (smoothed at 8 $\mathrm{mm}^{3}$ ) for each of the four conditions in every participant.

For both spatiotemporal and source level analyses, data were analyzed using a mass univariate general linear model method. We conducted a full factorial analysis using the following factors: Environment (stable and volatile) and PE (Standards and Deviants). We computed contrast images for main effects, interactions, and $t$ tests to gauge the differential effect between deviants and standards during stable and volatile conditions. In addition, we conducted multiple regression analyses with statistical learning error as the predictor and activity at the scalp and source level as the outcomes. Age was added into all models as a covariate, since attenuation in PE response occurs with age (Kiang et al., 2009). The order of volatile and stable conditions was also included as a covariate as it has been shown to influence PE responses (Todd et al., 2011, 2014). Finally, psychotic experience was added as a covariate to exclude any potential differences in volatile and stable conditions because of psychotic symptoms. All statistical maps are reported at a threshold of $p<0.05$ familywise error 


\section{Discovery Dataset}
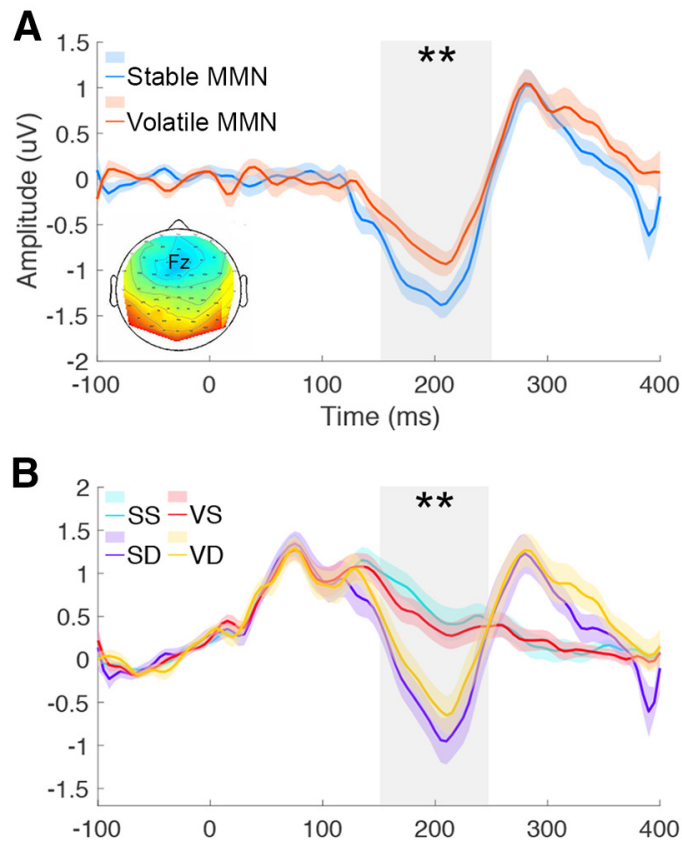

C

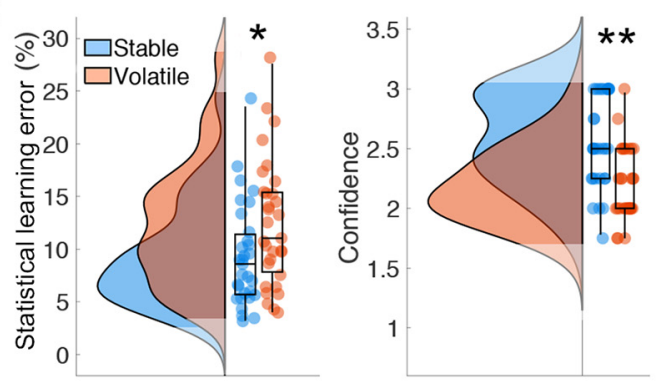

D

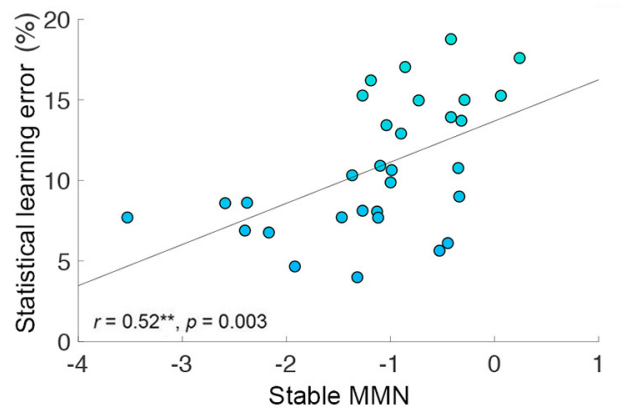

\section{Validation Dataset}

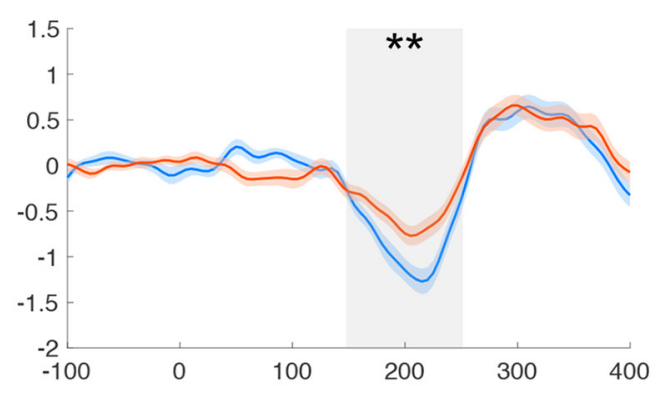

Replicated?
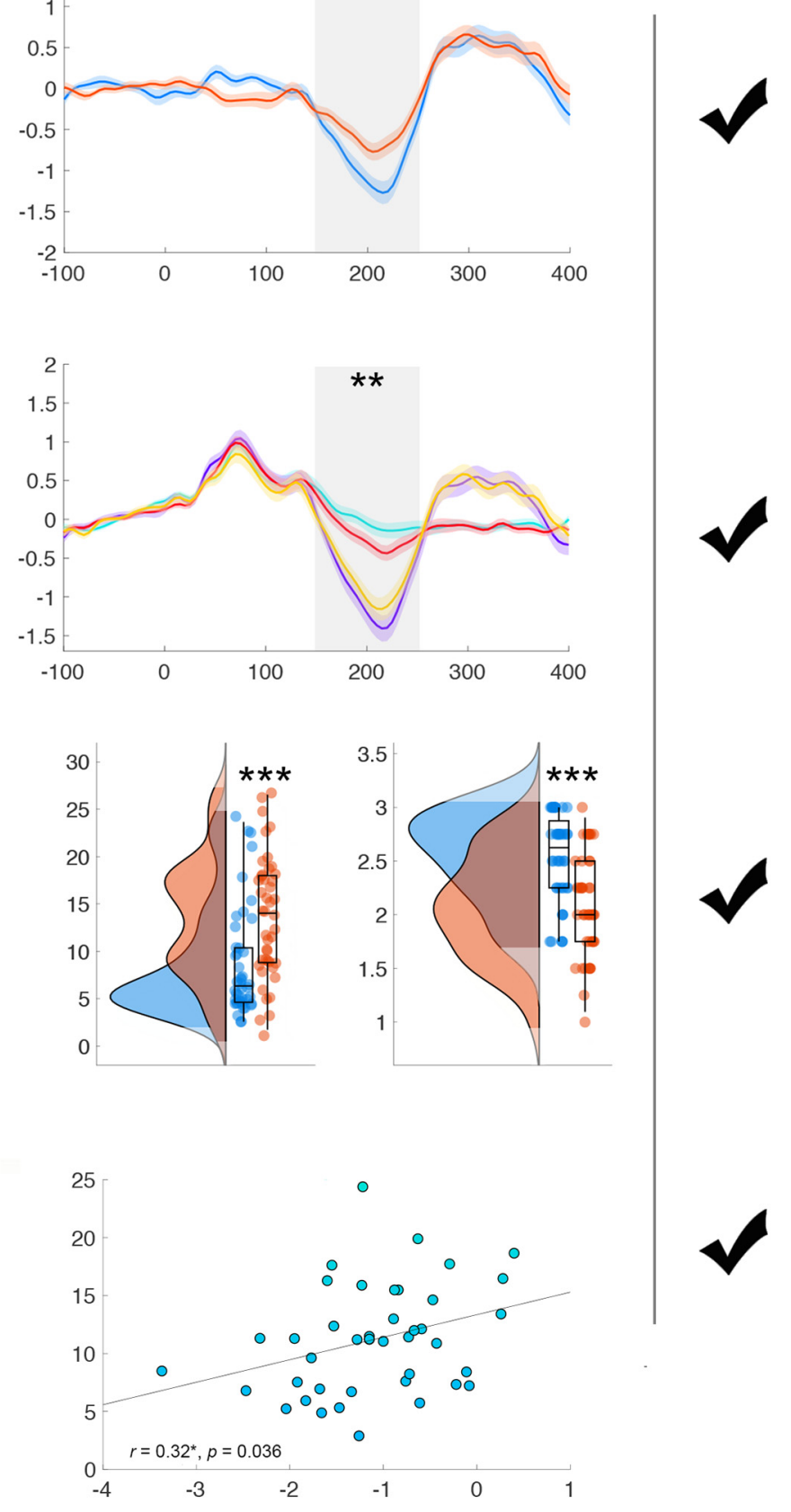

Figure 3. Prediction errors relate to statistical learning ability, indicating a precise prediction model in stable conditions. $A$, Significant interaction between volatility (stable - blue vs volatile orange) and MMN. B, Significant main effect of MMN, showing brain responses evoked by standards (green and red) and deviants (purple and yellow) in the context of stable and volatile conditions. C, Fewer statistical learning errors and greater confidence in stable (blue) than volatile (orange) conditions. The plots show individual data points, density plots and boxplots. D, Significant correlation between statistical learning errors and MMN in stable conditions. SS, Stable standard; SD, stable deviant; VS, volatile standard; VD, volatile deviant. ${ }^{*} p<0.05$; ${ }^{* *} p<0.001$, ${ }^{* * *} p<0.0001$. All of these findings were replicated across the Discovery and the Validation datasets.

(FWE) corrected for multiple comparisons for the spatiotemporal peak/volume or source region. We used the Porthole and Stormcloud toolbox from Taylor and Garrido (2020) to display the spatiotemporal figures and videos found in the Movie 1, Movie 2, Movie 3.

Dynamic causal modeling. Dynamic causal modeling (DCM) was used, which, similar to source reconstruction, also uses a spatial forward model. However, in addition to this, DCM incorporates a biologically informed temporal forward model, which places empirically derived constraints on the inversion and allows inferences about the source connectivity (Kiebel et al., 2009).

In the model specification, we defined the brain architecture based on previous robust findings (Opitz et al., 2002; Garrido et al., 2007), demonstrating that a three-level hierarchical brain model underlies the generation of PE responses evoked in auditory oddball paradigms. This 
Table 2. Pearson's and Bayesian correlation matrix for psychotic-like experience, statistical learning, and prediction error

\begin{tabular}{lllll}
\hline & & $\begin{array}{l}\text { Statistical } \\
\text { learning error }\end{array}$ & $\begin{array}{l}\text { Stable } \\
\text { prediction error }\end{array}$ & $\begin{array}{l}\text { Volatile } \\
\text { prediction error }\end{array}$ \\
\hline Discovery dataset & & & & \\
Psychotic-like experience & $r$ & $0.394^{*}$ & 0.326 & 0.188 \\
& $\mathrm{BF}_{10}$ & 4.370 & 1.986 & 0.609 \\
Statistical learning error & $r$ & & $0.522^{* *}$ & 0.327 \\
& $\mathrm{BF}_{10}$ & & $33.993^{* *}$ & 2.005 \\
Validation dataset & & & & \\
Psychotic-like experience & $r$ & $0.306^{*}$ & -0.001 & 0.024 \\
& $\mathrm{BF}_{10}$ & 2.553 & 0.191 & 0.217 \\
Statistical learning error & $r$ & & $0.324^{*}$ & 0.061 \\
& $\mathrm{BF}_{10}$ & & 3.137 & 0.267 \\
\hline
\end{tabular}

Table displays both Pearson's correlations $(r)$ and Bayes factors $\left(\mathrm{BF}_{10}\right)$. For all tests, the alternative hypothesis specifies that the correlation is positive $(+0)$

For Pearson's correlations: ${ }^{*} p<0.05,{ }^{* *} p<0.01$. For Bayes factors: ${ }^{*} \mathrm{BF}_{+0}>10,{ }^{*}{ }^{*} \mathrm{BF}+0>30$.

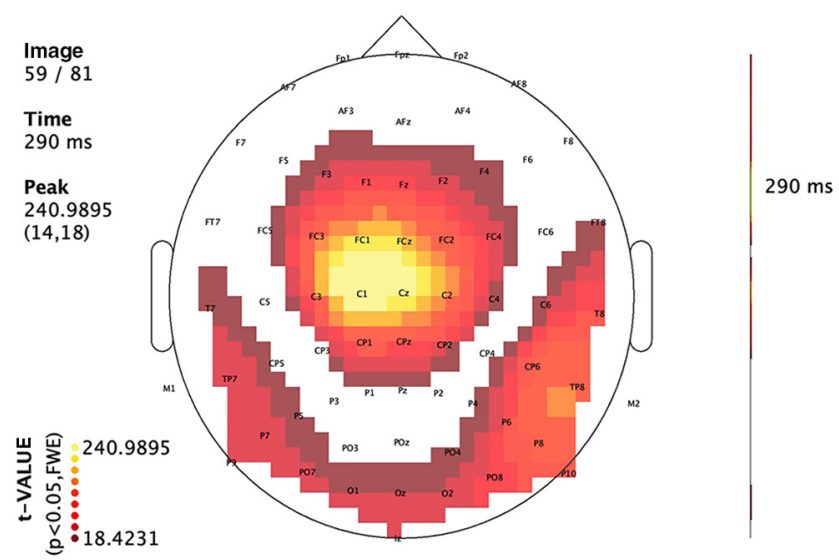

Movie 1. Spatiotemporal univariate analysis showing a significant main effect of PE in the Discovery dataset. [View online]

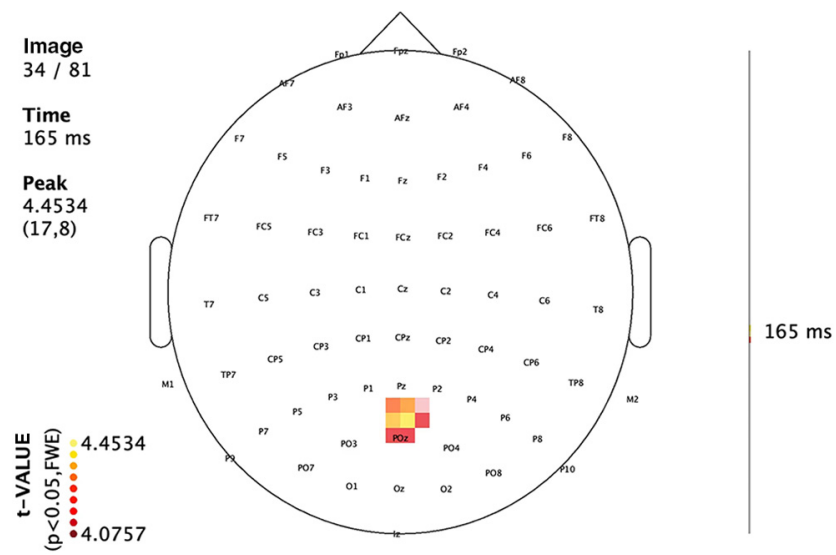

Movie 2. Spatiotemporal univariate analysis showing a significant interaction between PE and Volatility in the Discovery dataset. [View online]

model included the following: bilateral primary auditory cortex (A1; MNI coordinates: left, $[-42,-22,7]$; right, $[46,-14,8]$; chosen as the cortical input sources), bilateral superior temporal gyrus (STG; left $[-61,-32,8]$; right $[59,-25,8])$, and bilateral inferior frontal gyrus (IFG; left, [-46, 20, 8]; right, [46, 20, 8]). We considered nine competing model architectures that differed in source regions and the pattern of neuronal gain, and backward and forward connection (Extended Data

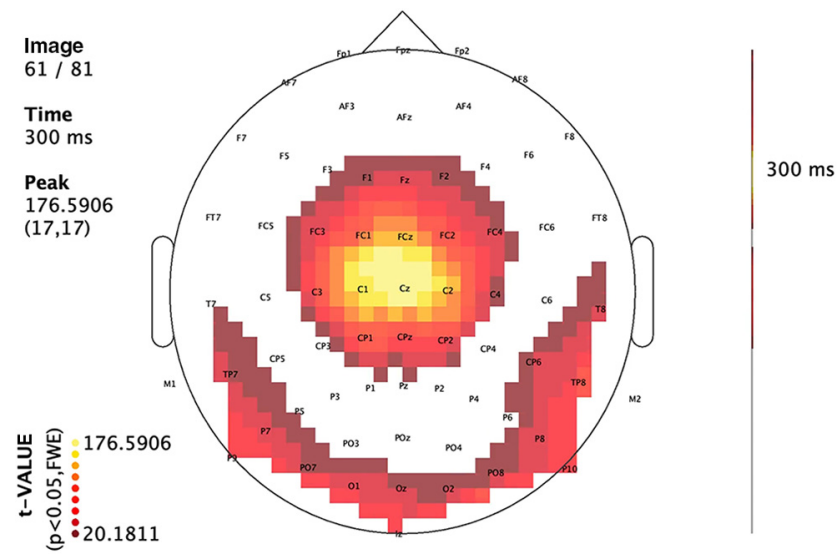

Movie 3. Spatiotemporal univariate analysis showing a significant main effect of PE in the Validation dataset. [View online]

Fig. 5-1). To model the effect of environment on effective connectivity, we examined the differences in PE response for stable versus volatile conditions. Specifically, we entered the stable PE responses (stable Deviants minus stable Standards) as "1" and volatile PE responses (volatile deviants minus volatile standards) as " 0 ." In this way, the connectivity estimates reflect the fact that stable PEs are the condition of interest and volatile PEs are the baseline comparison in DCM. The full details of the model specification have been described by Garrido, Friston (Garrido et al., 2008). Briefly, we modeled each source region with a single equivalent current dipole and an input time delay with a prior mean of $60 \mathrm{~ms}$; drift was modeled with a direct cosine transform of 1; and eight modes were selected for the $0-400 \mathrm{~ms}$ peristimulus time window, over stable PE and volatile PE trials.

The explanatory models were grouped by families based on the modulations placed on neuronal gain and extrinsic connectivity, as follows: (I) forward and neuronal gain family; (II) forward and backward family; (III) forward, backward, and neuronal gain family; and (IV) null family (no modulation in connectivity). We conducted Bayesian model selection, with a random-effects approach at both the model and family levels (62). We computed both expected and exceedance probabilities for the different families of models. The exceedance probability for a model indicates how well this model explains the data compared with the other models. We also conducted Bayesian model averaging to determine the strength of the effective connectivity at each connection, weighted by the likelihood of all models, and across all participants.

\section{Data availability}

The supporting data and materials for the article have been made available. Please find raw data files and the behavioral/connectivity scores at the following address: https://espace.library.uq.edu.au/view/UQ:724759.

\section{Results}

Our first aim was to compare the strength of the predictive models established in stable and volatile contexts. For this purpose, we examined the ERPs recorded at the frontocentral channel (Fz), namely the MMN. In line with the vast oddball literature, we consistently found that responses to deviant sounds were larger than responses elicited by standard sounds, regardless of the volatility for both the Discovery dataset $\left(F_{(1,30)}=45.33\right.$, $\left.p<0.001, \eta^{2}=0.60\right)$ and the Validation dataset $\left(F_{(1,40)}=88.14\right.$, $\left.p<0.001, \eta^{2}=0.69\right)$. Moreover, we found a significant interaction between MMN and volatility in the Discovery dataset $\left(F_{(1,30)}=11.06, p=0.002, \eta^{2}=0.27\right)$, which was replicated in the Validation dataset $\left(F_{(1,40)}=12.26, p=0.001, \eta^{2}=0.24\right)$. Critically, a follow-up analysis revealed that MMN was larger under the stable compared with the volatile conditions again for both the 


\section{Discovery Dataset}

\section{Validation Dataset}

A

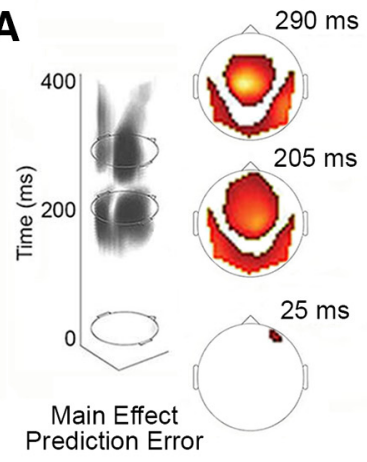

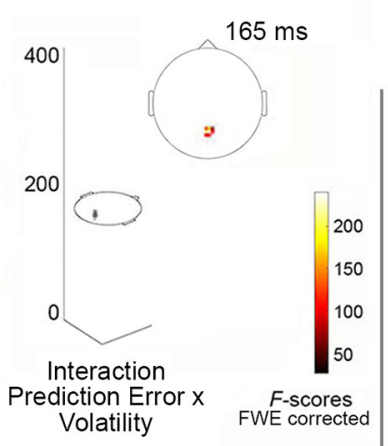
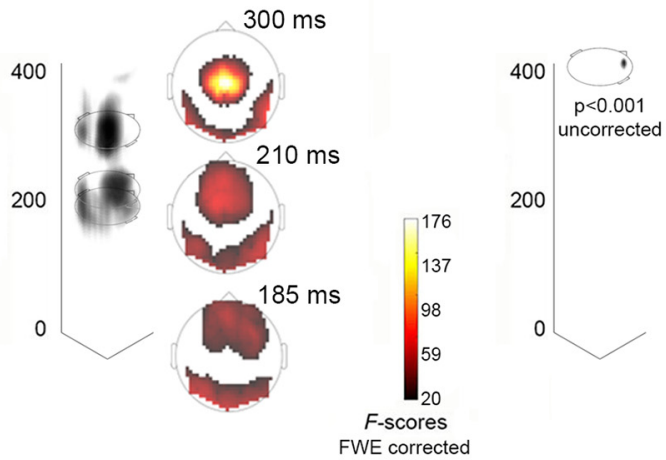

Replicated?

B

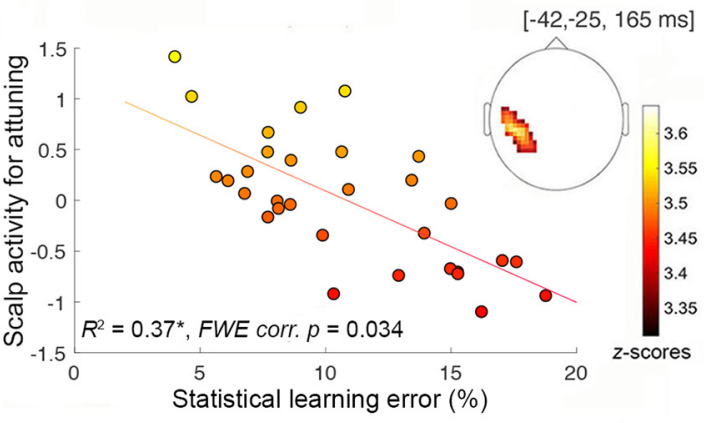

C

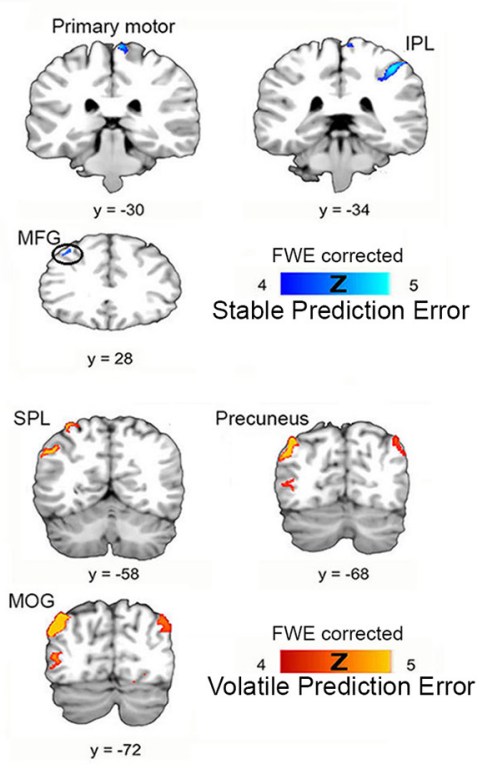

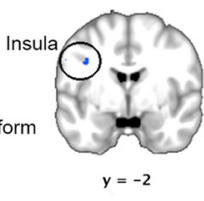

$p<0.001$ uncorrected

$\begin{array}{llll}2 & Z & 3\end{array}$

Stable Prediction Error
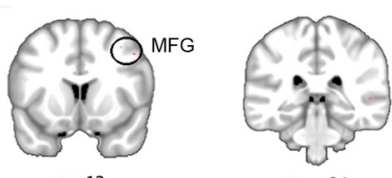

$y=12$

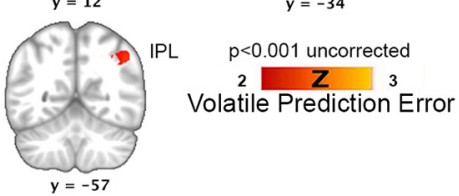

Figure 4. Brain responses underlying main effect of prediction error. $A$, Spatiotemporal univariate analysis revealed a significant main effect of PE (left column; this was replicated); and PE $\times$ volatility interaction (right column; this was not replicated). $\boldsymbol{B}$, Spatiotemporal multiple-regression analysis revealed a negative relationship between statistical learning errors and activity during the interaction (stable PE > volatile PE); however, this result was not replicated at FEW-corrected significance, even after a region of interest analysis. C, Source reconstruction analysis revealed significant clusters for stable PEs versus volatile PES; however, these results were not replicated. IPL, Inferior parietal lobule; MFG, middle frontal gyrus; SPL, superior parietal lobule; MOG, middle occipital gyrus. Except where otherwise specified, all maps are displayed at $p<0.05$, FWE whole-volume corrected (Movie 1, Movie 2, Movie 3, Extended Data Figure 4-1). ${ }^{*} p<0.05$.

Discovery set $\left(t_{(30)}=-3.33, p=0.002, d=-0.60\right)$ and the Validation set $\left(t_{(40)}=-3.74, p=0.001, d=-0.61\right.$; Fig. $\left.3 A, B\right)$.

We next examined differences in mean percentage errors in probability estimation (statistical learning error) and mean confidence ratings during stable and volatile conditions. The data showed that participants had fewer errors in statistical learning during stable conditions (Discovery: mean $=9.30 \%$, $\mathrm{SE}=0.87$;
Validation: mean $=8.62 \%, \mathrm{SE}=0.87)$, compared with volatile conditions (Discovery: mean $=12.38 \%, \mathrm{SE}=1.07$; Validation: mean $=13.54 \%, \mathrm{SE}=0.97$; Discovery: $t_{(30)}=-2.40, p=0.023, d=$ -0.43 ; Validation: $\left.t_{(43)}=-4.21, p<0.0001, d=-0.81\right)$. In addition, participants had greater confidence in their probability estimates during stable conditions (Discovery: mean $=2.57, \mathrm{SE}=0.07$; Validation: mean $=2.53, \mathrm{SE}=0.06)$, compared with volatile 


\section{Discovery Dataset}
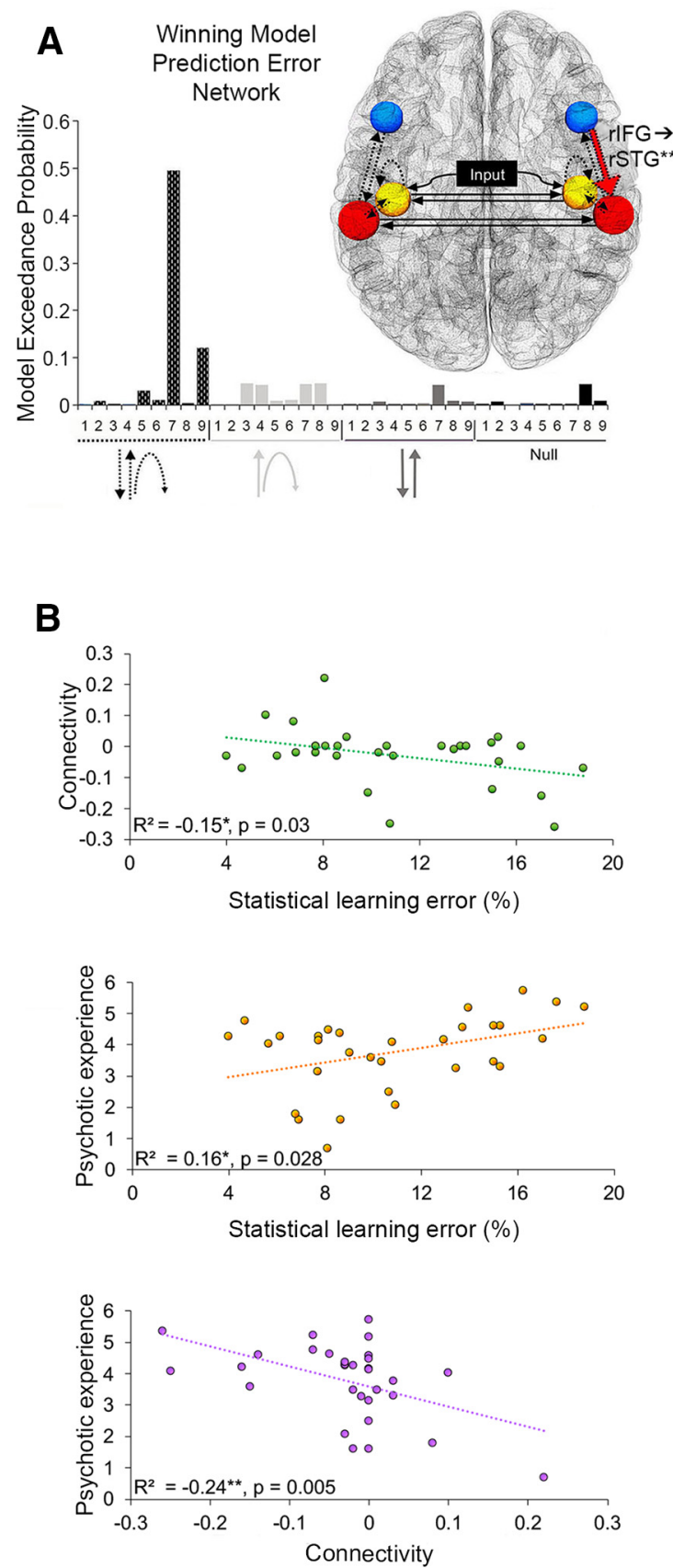

\section{Validation Dataset}

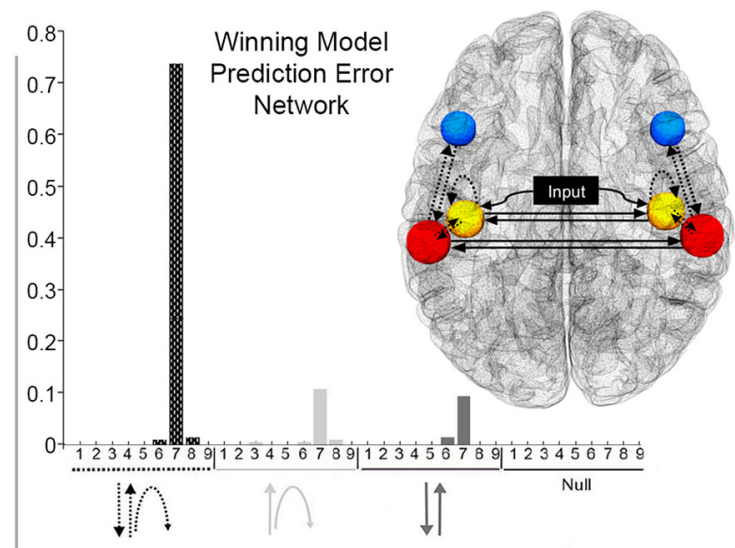

Replicated?
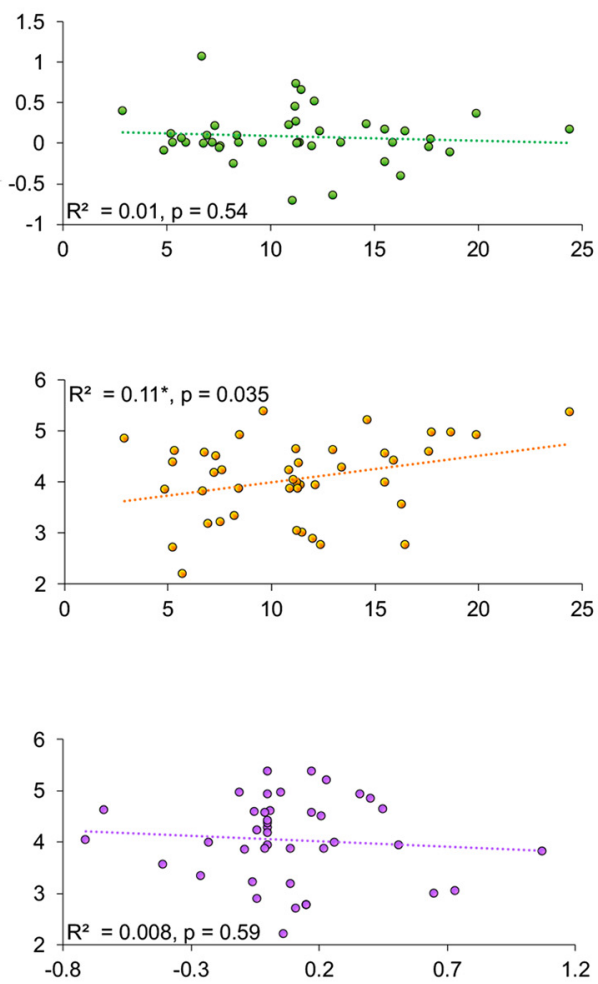

Figure 5. Statistical learning errors predict the severity of psychotic-like experiences in the nonclinical population. $\boldsymbol{A}$, The winning model architecture, replicated across both datasets, had connections between all six regions bilaterally (model 7), included volatility-dependent modulations in neuronal gain, and forward and backward connections. $\boldsymbol{B}$, Regression plots for statistical learning errors and top-down frontotemporal connectivity (in green; not replicated); statistical learning errors and psychotic-like experiences (in orange; replicated); and top-down frontotemporal connectivity and psychotic experiences (in purple; not replicated; Extended Data Figure 5-1). ${ }^{*} p<0.05 ;{ }^{* *} p<0.001$

conditions (Discovery: mean $=2.19, \mathrm{SE}=0.05$; Validation: mean $=2.08, \quad \mathrm{SE}=0.07 ;$ Discovery: $t_{(30)}=4.78, p<0.001, d=0.86$; Validation: $t_{(43)}=6.98, p<0.0001, d=1.00$; Fig. $3 C$ ).

We then asked whether statistical learning errors were related to the degree of MMN response. Pearson's correlations and Bayesian analysis revealed a significant correlation between statistical learning errors and MMN in stable conditions [Discovery: $p=0.003$ ( $\left.p_{\text {adjusted }}<0.01\right) ; \mathrm{BF}_{+0}=33.99$; Validation: $p=0.036$,
$\mathrm{BF}_{+0}=3.14$; Fig. $3 D$, Table 2], which had very strong evidence in the Discovery dataset and moderate evidence in the Validation dataset.

Next, we asked whether aberrations in behavior (greater statistical learning errors) and neurophysiology (attenuated MMN) were also aligned on the psychosis continuum. Pearson's and Bayesian correlations were conducted on psychotic experiences, statistical learning errors, as well as MMN in stable and volatile 
conditions. We found a significant correlation between psychotic experience and errors in statistical learning that was replicated across both datasets (Discovery: $p=0.028, \mathrm{BF}_{+0}=4.37$; Validation: $p=0.035, \mathrm{BF}_{+0}=2.55$; Table 2). This showed that healthy individuals with greater psychotic experiences were worse at learning about sensory regularities. The fact that this was replicated provides strong support for behavioral alterations in sensory inference aligning on the nonclinical end of the psychosis continuum.

To further investigate the sensory PEs evoked by regularity violations with fewer spatial and temporal constraints, we ran a general linear model for the whole spatiotemporal volume of brain activity. First, we supported previous auditory oddball findings by showing a significant main effect of $\mathrm{PE}$ response (standard sounds vs deviant sounds) peaking at $290 \mathrm{~ms}$ (peaklevel $F=240.99, p_{\text {FWE }}<0.001$ ) and $205 \mathrm{~ms}$ (peak-level $F=$ $\left.170.92, p_{\mathrm{FWE}}<0.001\right)$ in central and occipitoparietal channels, and $25 \mathrm{~ms}$ (peak-level $F=25.26, p_{\mathrm{FWE}}=0.004$ ) in frontal channels (Movie 1). We also replicated our own findings by showing the main effects of PE response in the Validation dataset that peaked at similar time points, as follows: $300 \mathrm{~ms}$ (peak-level $\left.F=176.59, p_{\text {FWE }}<0.001\right), 210 \mathrm{~ms}$ (peak-level $F=77.44, p_{\text {FWE }}<$ 0.001 ), and $185 \mathrm{~ms}$ (peak-level $F=68.76, p_{\mathrm{FWE}}<0.001$ ) in central and occipitoparietal channels (note: $25 \mathrm{~ms}$ was not replicated; Movie 3). We originally found a significant interaction between $\mathrm{PE}$ response and volatility, at $165 \mathrm{~ms}$ over occipitocentral channels (peak-level $z=4.27, p_{\mathrm{FWE}}=0.015$; Fig. $4 A$, Movie 2); however, this did not replicate in the Validation set.

Next, we asked whether statistical learning errors were related to the neuronal correlates of volatility attuning. To address this question, we conducted a spatiotemporal multiple-regression analysis of the interaction between PEs and volatility (stable PEs $>$ volatile PEs) with statistical learning error as the predictor variable. Our data in the Discovery study showed that a decrease in statistical learning errors significantly predicted an increase in brain activity at $165 \mathrm{~ms}$ (peak-level $z=3.64$, cluster-level $p_{\mathrm{FWE}}=$ 0.034; Fig. 4B). To determine where in the brain this effect came from, we used source reconstruction techniques, which uncovered an increased activity in the right superior frontal gyrus (peak-level $z=2.19, p_{\text {uncorrected }}=0.014$ ) and the right fusiform gyrus (peak-level $z=1.89, p_{\text {uncorrected }}=0.029$; Extended Data Fig. 4-1). However, these results did not replicate, even after conducting a spatiotemporal volume of interest analysis (based on the result from the Discovery study) for the multiple regression.

In the Discovery study, source-level analysis revealed that stable PEs engaged the middle frontal gyrus (peak-level $z=4.23$, $p_{\mathrm{FWE}}=0.02$ ), primary motor area (peak-level $z=4.45, p_{\mathrm{FWE}}=$ 0.009 ), and inferior parietal lobule (peak-level $z=4.56, p_{\mathrm{FWE}}=$ 0.006). In comparison, volatile PEs engaged precuneus (peaklevel $z=5.05, p_{\mathrm{FWE}}=0.001$ ) and middle occipital gyrus (peaklevel $z=4.39, p_{\mathrm{FWE}}=0.011$ ). However, we were not able to replicate these findings in the independent Validation sample. None of the voxels survived FWE correction, nor did the uncorrected clusters correspond with the Discovery study (Fig. 4C).

The network architecture underlying the PE response has been extensively studied previously (Opitz et al., 2002; Garrido et al., 2007). Here, we were interested in (1) the effect of contextual volatility on neuronal PE responses and (2) the effective connectivity underpinning psychotic traits and statistical learning.

Bayesian model comparison was performed on 36 different dynamic causal models (Extended Data Fig. 5-1), which were based on the functional brain architecture previously shown to underlie PE responses (Opitz et al., 2002; Garrido et al., 2007). Here, PEs in stable blocks were compared with those in volatile
Table 3. Pearson's and Bayesian correlation matrix for psychotic-like experience and effective connections

\begin{tabular}{|c|c|c|c|c|}
\hline & \multicolumn{4}{|c|}{ Psychotic-like experience } \\
\hline & \multicolumn{2}{|c|}{ Discovery dataset } & \multicolumn{2}{|c|}{ Validation dataset } \\
\hline & $r$ & $\mathrm{BF}_{10}$ & $r$ & $\mathrm{BF}_{10}$ \\
\hline$|A 1-| A \mid$ & 0.225 & 0.108 & 0.202 & 0.091 \\
\hline rA1-rA1 & -0.094 & 0.346 & -0.253 & 1.258 \\
\hline IA1-ISTG1 & 0.186 & 0.12 & -0.094 & 0.328 \\
\hline ISTG1-IIFG & -0.118 & 0.395 & -0.063 & 0.271 \\
\hline rA1-rSTG1 & 0.074 & 0.169 & 0.192 & 0.094 \\
\hline rSTG1-rIFG & 0.143 & 0.135 & 0.141 & 0.11 \\
\hline ISTG1-IA1 & 0.136 & 0.138 & 0.197 & 0.093 \\
\hline IIFG-ISTG1 & 0.219 & 0.11 & -0.055 & 0.26 \\
\hline rSTG1-rA1 & -0.076 & 0.316 & -0.244 & 1.142 \\
\hline rIFG-rSTG & $-0.489^{* *}$ & $18.282^{*}$ & -0.088 & 0.316 \\
\hline
\end{tabular}

Pearson's correlations, $r$; Bayes factors, $\mathrm{BF}_{10} ; \mathrm{I}$, left; $r$, right. For all tests, the alternative hypothesis specifies that the correlation is negative $\left(_{-0}\right)$.

For Pearson's correlations: ${ }^{*} p<0.05,{ }^{* *} p<0.01$. For Bayes factors: ${ }^{*} \mathrm{BF}_{-0}>10,{ }^{* *} \mathrm{BF}_{-0}>30$.

blocks. Results from Bayesian model selection using randomeffects family-level analysis (in both the Discovery and Validation datasets) indicated that the best model included connections among six a priori defined regions, with inputs to left and right $A 1$, neuronal gain within the $A 1$, bilateral connections between A1 and STGs and between STGs and IFGs, as well as lateral connections between left and right $\mathrm{A} 1$, and left and right STG (model 7; Fig. 5A). The optimal model, which had greater modulation in neuronal gain, as well as backward and forward connections, was replicated across both the Discovery and the Validation datasets (Fig. 5A).

Next, we examined the altered neural dynamics related to behavior and psychotic-like traits in the general, nonclinical population. First, we examined brain connectivity estimates by applying Bayesian model averaging across all models (weighted by their probability) and participants. In the Discovery dataset, we found a strong, significant negative correlation between psychotic-like experiences and top-down connectivity from the right IFG to STG $\left[p=0.005\right.$ ( $p_{\text {adjusted }}<0.05$ ), $\mathrm{BF}_{-0}=18.28$; Fig. $5 B$, Table 3], and between statistical learning errors and top-down connectivity from the right IFG to STG ( $p=0.03$; Fig. $5 B)$. However, these findings were not replicated.

\section{Discussion}

The aims of the current study were to investigate and replicate the neural mechanisms that underpin statistical learning and volatility attuning in healthy individuals with a range of psychoticlike experiences. We measured individuals' statistical learning abilities (by asking them to estimate the probabilities of sounds) in stable and volatile conditions while recording their brain activity using EEG. We pursued these goals in two independent datasets and in turn discuss the findings that did replicate across both. During stable conditions, compared with volatile conditions, statistical learning improved, prediction errors increased, and there was greater modulation of neuronal gain, forward connections, and backward connections. For the first time, we showed that statistical learning (behaviorally assessed) relates to prediction errors. Moreover, we were able to replicate the finding that a greater degree of psychotic-like experiences in healthy individuals is associated with impaired sensory statistical learning ability, providing strong evidence for the existence of a continuum of psychosis in the nonclinical population. 
MMN responses, statistical learning, and confidence, were enhanced in stable, more predictable environments, than in more volatile, less predictable, environments. Increased $\mathrm{MMN}$ in stable (compared with volatile) conditions has been identified in previous studies (Todd et al., 2011, 2014). MMN signals a PE: a violation between what the brain predicts will happen and what is actually experienced. As such, PEs are fundamental teaching signals that drive updating of the brain's predictive model of the sensed world (Rao and Ballard, 1999; Garrido et al., 2009a). The amount of updating depends on the precision weighting of the PEs; which in turn depends on the relative uncertainty of sensory information and prior beliefs (Mathys et al., 2011). In Bayesian generative models, such as the Hierarchical Gaussian Filter, environmental volatility is associated with decreased sensory precision resulting in reduced precision weighting of the PEs (Mathys et al., 2014). Thus, it follows that PE responses to regularity violations will be attenuated in volatile compared with stable conditions (Garrido et al., 2009b), which is in line with our findings.

MMN in stable conditions were found to be associated with better sensory statistical learning in the current study. Statistical learning is the process by which the brain learns the statistical structure in the environment and forms predictive models of what is likely to happen next (Bendixen et al., 2007; Dale et al., 2012; Tavano et al., 2014). Previous studies have demonstrated that individuals are able to implicitly learn the statistical structure of sensory events in the environment (Garrido et al., 2013; Lecaignard et al., 2015). Crucially, by simultaneously recording $\mathrm{PE}$ responses (i.e., MMN) and behaviorally measuring statistical learning, we showed, for the first time and across both the Discovery and the Validation samples, that greater sensory PEs are associated with improved explicit ability to gauge the sensory regularities within one's environment. This was specifically found for MMN in stable rather than volatile conditions, suggesting that MMN may not relate to the long-term rule updates (i.e., global regularities) that influence statistical learning in the volatile conditions (Mathys et al., 2011).

At the neural level, we found differences in the pattern of connections underlying PE response in stable compared with volatile conditions. Larger PEs in stable compared with volatile conditions were produced by enhanced modulations in neuronal gain, and backward and forward connections. Forward and backward connections are thought to convey PEs and predictions (i.e., beliefs about sensory input), respectively. Neuronal gain emulates local adaptation of neuronal responses (Kiebel et al., 2007). This finding is in keeping with the predictive coding account of the mechanisms underlying the perception of an auditory oddball sequence (Garrido et al., 2008), and suggest that more precise predictive models about sensory input are enabled by greater brain connectivity in stable than in volatile PEs.

We did not find consistent associations between alterations in the neurophysiology or brain connectivity and psychotic-like experiences in our nonclinical samples. While in our Discovery sample we found weaker top-down frontotemporal connectivity in people who reported more psychotic-like experiences, we failed to replicate this finding in the Validation sample. It is possible that these brain alterations are not as robust in the healthy population that experiences mild, psychotic-like experiences. Indeed, there is conflicting evidence regarding the attenuation of PEs (e.g., MMN and P300) in the nonclinical psychosis continuum. A recent study has found reductions in sensory PEs (Oestreich et al., 2019), while others have reported a lack of/ mixed evidence for an association (Klein et al., 1999; Sumich et al., 2014; Broyd et al., 2016). Further research looking at the full continuum, including a range of psychiatric groups, may elucidate whether or not a relationship between psychotic experiences and brain alterations exists, and, if so, whether it also extends into the nonclinical, healthy population.

Critically, in our study we uncovered and replicated the behavioral aberrations underlying predictive processing that are aligned on the nonclinical continuum of psychosis. Previous research has also uncovered altered behavior, for example in social inference (Wellstein et al., 2020), self-tickling (Lemaitre et al., 2016), and force matching (Teufel et al., 2010), in individuals with subclinical psychotic experiences. However, a study by Humpston et al. (2017), investigating a range of predictive processing behaviors, such as force matching, associative learning, and reversal learning, was not been able to replicate the relationship between altered behavior and psychotic-like experiences in the general population. In response to the recent replication crisis in the field, we decided to collect two independent samples for discovery and validation of our findings. Indeed, we were able to replicate that psychotic-like experiences are associated with impaired statistical learning behavior. Therefore, we show, with strengthened credibility, that there exists a continuum of psychosis, even in the nonclinical end of the spectrum, that manifests on a declining ability in sensory statistical learning.

In the current study, we explored the brain dynamics underpinning sensory learning under uncertainty, as well as the relationship between disruptions to predictive processes and psychotic-like experiences in healthy individuals. We originally investigated these processes in a Discovery dataset and then sought to replicate the findings in an independent Validation dataset. We found that individuals learn better and their brain $\mathrm{PE}$ responses are greater during stable than during volatile conditions. There is greater modulation of neuronal gain, and forward and backward connections in stable conditions when a more precise model of the sensed environment is represented in the brain. In addition, we were able to show that individuals with stronger stable PE responses had improved sensory statistical learning behavior. Importantly, we were able to replicate that aberrations in predictive processes are aligned on a nonclinical continuum of psychosis, in the sense that healthy people with more psychoticlike experiences have poorer statistical learning ability.

\section{References}

Adams RA, Stephan KE, Brown HR, Frith CD, Friston KJ (2013) The computational anatomy of psychosis. Front Psychiatry 4:47.

Adams RA, Huys QJ, Roiser JP (2016) Computational Psychiatry: towards a mathematically informed understanding of mental illness. J Neurol Neurosurg Psychiatry 87:53-63.

Angela Y, Dayan P (2002) Expected and unexpected uncertainty: ACh and $\mathrm{NE}$ in the Neocortex. Proceedings of the 15th International Conference on Neural Information Processing Systems, pp. 173-180. MIT Press. Cambridge:MA.

Behrens TE, Woolrich MW, Walton ME, Rushworth MF (2007) Learning the value of information in an uncertain world. Nat Neurosci 10:12141221.

Bendixen A, Roeber U, Schröger E (2007) Regularity extraction and application in dynamic auditory stimulus sequences. J Cogn Neurosci 19:16641677.

Broyd SJ, Michie PT, Bruggemann J, van Hell HH, Greenwood L-m, Croft RJ, Todd J, Lenroot R, Solowij N (2016) Schizotypy and auditory mismatch negativity in a non-clinical sample of young adults. Psychiatry Res Neuroimaging 254:83-91.

Dale R, Duran ND, Morehead JR (2012) Prediction during statistical learning, and implications for the implicit/explicit divide. Adv Cogn Psychol 8:196-209. 
Friston K, Harrison L, Daunizeau J, Kiebel S, Phillips C, Trujillo-Barreto N, Henson R, Flandin G, Mattout J (2008) Multiple sparse priors for the M/ EEG inverse problem. Neuroimage 39:1104-1120.

Garrido MI, Kilner JM, Kiebel SJ, Stephan KE, Friston KJ (2007) Dynamic causal modelling of evoked potentials: a reproducibility study. Neuroimage 36:571-580

Garrido MI, Friston KJ, Kiebel SJ, Stephan KE, Baldeweg T, Kilner JM (2008) The functional anatomy of the MMN: a DCM study of the roving paradigm. Neuroimage 42:936-944.

Garrido MI, Kilner JM, Stephan KE, Friston KJ (2009a) The mismatch negativity: a review of underlying mechanisms. Clin Neurophysiol 120:453463

Garrido MI, Kilner JM, Kiebel SJ, Friston KJ (2009b) Dynamic causal modeling of the response to frequency deviants. J Neurophysiol 101:2620-2631.

Garrido MI, Sahani M, Dolan RJ (2013) Outlier responses reflect sensitivity to statistical structure in the human brain. PLoS Comput Biol 9: e1002999.

Gronau QF, Ly A, Wagenmakers E-J (2020) Informed Bayesian t-tests. Am Stat 74:137-143

Humpston CS, Evans LH, Teufel C, Ihssen N, Linden DEJ (2017) Evidence of absence: no relationship between behaviourally measured prediction error response and schizotypy. Cogn Neuropsychiatry 22:373-390.

Jang K-M, Kim M-S (2014) Relationships among event-related potentials, memory, and schizophrenic symptoms in college students with schizotypal-traits. Open J Psychiatr 4:353-363.

Kiang M, Braff DL, Sprock J, Light GA (2009) The relationship between preattentive sensory processing deficits and age in schizophrenia patients. Clin Neurophysiol 120:1949-1957.

Kiebel SJ, Garrido MI, Friston KJ (2007) Dynamic causal modelling of evoked responses: the role of intrinsic connections. Neuroimage 36:332345

Kiebel SJ, Garrido MI, Moran R, Chen CC, Friston KJ (2009) Dynamic causal modeling for EEG and MEG. Hum Brain Mapp 30:1866-1876.

Klein C, Berg P, Rockstroh B, Andresen B (1999) Topography of the auditory P300 in schizotypal personality. Biol Psychiatry 45:1612-1621.

Lecaignard F, Bertrand O, Gimenez G, Mattout J, Caclin A (2015) Implicit learning of predictable sound sequences modulates human brain responses at different levels of the auditory hierarchy. Front Hum Neurosci 9:505.

Lemaitre A-L, Luyat M, Lafargue G (2016) Individuals with pronounced schizotypal traits are particularly successful in tickling themselves. Conscious Cogn 41:64-71.

Litvak V, Friston K (2008) Electromagnetic source reconstruction for group studies. Neuroimage 42:1490-1498.

Loewy RL, Bearden CE, Johnson JK, Raine A, Cannon TD (2005) The prodromal questionnaire (PQ): preliminary validation of a self-report screening measure for prodromal and psychotic syndromes. Schizophr Res 79:117-125.

Mathys C, Daunizeau J, Friston KJ, Stephan KE (2011) A Bayesian foundation for individual learning under uncertainty. Front Hum Neurosci 5:39.

Mathys CD, Lomakina EI, Daunizeau J, Iglesias S, Brodersen KH, Friston KJ, Stephan KE (2014) Uncertainty in perception and the hierarchical Gaussian filter. Front Hum Neurosci 8:825.

Näätänen R, Shiga T, Asano S, Yabe H (2015) Mismatch negativity (MMN) deficiency: a break-through biomarker in predicting psychosis onset. Int J Psychophysiol 95:338-344.

Oestreich LKL, Randeniya R, Garrido MI (2019) Auditory prediction errors and auditory white matter microstructure associated with psychotic-like experiences in healthy individuals. Brain Struct Funct 224:3277-3289.

Oostenveld R, Praamstra P (2001) The five percent electrode system for high-resolution EEG and ERP measurements. Clin Neurophysiol 112:713-719.

Opitz B, Rinne T, Mecklinger A, von Cramon DY, Schröger E (2002) Differential contribution of frontal and temporal cortices to auditory change detection: fMRI and ERP results. Neuroimage 15:167-174.
Randeniya R, Oestreich LKL, Garrido MI (2018) Sensory prediction errors in the continuum of psychosis. Schizophr Res 191:109-122.

Rao RPN, Ballard DH (1999) Predictive coding in the visual cortex: a functional interpretation of some extra-classical receptive-field effects. Nat Neurosci 2:79-87.

Schwartenbeck P, FitzGerald T, Dolan RJ, Friston K (2013) Exploration, novelty, surprise, and free energy minimization. Front Psychol 4:710.

Sherman MT, Seth AK, Barrett AB, Kanai R (2015) Prior expectations facilitate metacognition for perceptual decision. Conscious Cogn 35:53-65.

Sidak Z (1967) Rectangular confidence regions for the means of multivariate normal distributions. J Am Stat Assoc 62:626-633.

Southwell R, Chait M (2018) Enhanced deviant responses in patterned relative to random sound sequences. Cortex 109:92-103.

Squires NK, Squires KC, Hillyard SA (1975) Two varieties of long-latency positive waves evoked by unpredictable auditory stimuli in man. Electroencephalogr Clin Neurophysiol 38:387-401.

Sterzer P, Adams RA, Fletcher P, Frith C, Lawrie SM, Muckli L, Petrovic P, Uhlhaas P, Voss M, Corlett PR (2018) The predictive coding account of psychosis. Biol Psychiatry 84:634-643.

Sumich A, Castro A, Kumari V (2014) N100 and N200, but not P300, amplitudes predict paranoia/suspiciousness in the general population. Pers Individ Dif 61-62:74-79.

Tavano A, Widmann A, Bendixen A, Trujillo-Barreto N, Schröger E (2014) Temporal regularity facilitates higher-order sensory predictions in fast auditory sequences. Eur J Neurosci 39:308-318.

Taylor JA, Garrido MI (2020) Porthole and Stormcloud: tools for visualisation of spatiotemporal M/EEG statistics. Neuroinformatics 18:351-363.

Teufel C, Kingdon A, Ingram JN, Wolpert DM, Fletcher PC (2010) Deficits in sensory prediction are related to delusional ideation in healthy individuals. Neuropsychologia 48:4169-4172.

Todd J, Michie PT, Schall U, Karayanidis F, Yabe H, Näätänen R (2008) Deviant matters: duration, frequency, and intensity deviants reveal different patterns of mismatch negativity reduction in early and late schizophrenia. Biol Psychiatry 63:58-64.

Todd J, Provost A, Cooper G (2011) Lasting first impressions: a conservative bias in automatic filters of the acoustic environment. Neuropsychologia 49:3399-3405.

Todd J, Heathcote A, Whitson LR, Mullens D, Provost A, Winkler I (2014) Mismatch negativity (MMN) to pitch change is susceptible to order-dependent bias. Front Neurosci 8:180.

van Os J, Reininghaus U (2016) Psychosis as a transdiagnostic and extended phenotype in the general population. World Psychiatry 15:118-124.

Vossel S, Mathys C, Daunizeau J, Bauer M, Driver J, Friston KJ, Stephan KE (2014) Spatial attention, precision, and Bayesian inference: a study of saccadic response speed. Cereb Cortex 24:1436-1450.

Wacongne C, Changeux JP, Dehaene S (2012) A neuronal model of predictive coding accounting for the mismatch negativity. J Neurosci 32:36653678.

Wagenmakers EJ, Marsman M, Jamil T, Ly A, Verhagen J, Love J, Selker R, Gronau QF, Šmíra M, Epskamp S, Matzke D, Rouder JN, Morey RD (2017) Bayesian inference for psychology. Part I: Theoretical advantages and practical ramifications. Psychon Bull Rev 25:35-57.

Weber L, Diaconescu A, Tomiello S, Schöbi D, Iglesias S, Mathys C, Haker H, Stefanics G, Schmidt A, Kometer M, Vollenweider FX, Stephan KE (2018) F157. Hierarchical prediction errors during auditory mismatch under pharmacological manipulations: a computational single-trial EEG analysis. Schizophr Bull 44(Suppl 1):S281-S282.

Weber LA, Diaconescu AO, Mathys C, Schmidt A, Kometer M, Vollenweider F, Stephan KE (2020) Ketamine affects prediction errors about statistical regularities: a computational single-trial analysis of the mismatch negativity. J Neurosci 40:5658-5668.

Wellstein KV, Diaconescu AO, Bischof M, Rüesch A, Paolini G, Aponte EA, Ullrich J, Stephan KE (2020) Inflexible social inference in individuals with subclinical persecutory delusional tendencies. Schizophr Res 215:344-351. 